\title{
Treatment of Parkinsonism with Laevo-Dopa
}

\author{
C. MAWDSLEY,* M.B., F.R.C.P.ED., M.R.C.P
}

\begin{abstract}
Cummary: Thirty-two patients with Parkinsonism were $\checkmark$ treated with L-dopa. Nine were markedly and 14 moderately improved. Twenty patients tolerated the drug well, derived definite improvement, and were treated for an average period of six months. Improvement was sustained during this period even though the average daily dosage of L-dopa was reduced. Dose-dependent sideeffects occurred in 25 patients. It is suggested that dosage schedules should be flexible and tailored to the needs of each individual patient, and that treatment should be continued for six months before presuming it to be ineffective.

It is concluded that L-dopa often ameliorates Parkinsonism for long periods, but its effect on the natural history of the disease is undetermined.
\end{abstract}

\section{Introduction}

Dopamine is widely distributed in the body, and its varying concentrations in the tissues reflect its usual role as a precursor of noradrenaline. The caudate nucleus and putamen, however, are rich in dopamine (Carlsson, 1959), though these structures contain little noradrenaline (Vogt, 1954). This discrepancy suggested that dopamine in this region might itself be active physiologically. Ehringer and Hornykiewicz (1960) showed that the dopamine content of the caudate nucleus and putamen was greatly reduced in post-mortem specimens taken from patients with Parkinsonism.

Trétiakoff (1919) first suggested that the principal lesion causing Parkinsonism lay in the substantia nigra. This hypothesis was supported by Hassler (1938). Many fibres run from the substantia nigra to the pallidum (Carpenter and McMasters, 1964), though few, if any, run in the reverse direction. Efferent fibres from both parts of the striatum, the putamen and caudate nuclei, communicate with the globus pallidus, and a direct connexion between the striatum and the substantia nigra has been defined (Rundles and Papez, 1937).

Bloom et al. (1965) showed that dopamine inhibits the firing of cells in the caudate nucleus and that acetylcholine increases the firing rate.

These biochemical and anatomical studies do not completely clarify the physiological defects which result in the syndrome of Parkinsonism, but they do indicate a rational approach. They suggest that between the substantia nigra, the pallidum, and the striatum acetylcholine is an excitatory transmitter, while dopamine there acts in an inhibitory fashion. It may be further suggested that in Parkinsonism there is an imbalance between these two transmitter systems, with cholinergic activity relatively increased and the activity of dopaminergic neurones relatively reduced. The beneficial effects of the long-established anti-Parkinsonian drugs could thus be explained by their central anticholinergic action. This theory does not necessarily imply that Parkinsonism is due to purely biochemical defects, since lower dopamine levels in the

*Senior Lecturer in Neurology, University of Edinburgh; Consultant Neurologist, Royal Infirmary, and Northern General Hospital, Edinburgh. striatum could well be secondary to cellular loss; nor does it exclude the possibility that other agents, such as noradrenaline, may play some part in modifying the functions of the basal ganglia. It does, however, suggest that Parkinsonism might be helped by enhancing the activity of the dopaminergic system. These considerations prompted attempts to augment the dopamine levels in the striatum. Dopamine does not penetrate the blood-brain barrier, and hence its precursor dihydroxyphenylalanine (dopa), was the agent employed.

Initial reports by Birkmayer and Hornykiewicz (1961) and Barbeau (1962) indicated that intravenous or oral dopa was beneficial in Parkinsonism. Later work (Greer and Williams, 1963; Fehling, 1966; Rinne and Sonninen, 1968) cast doubt on these earlier findings. Cotzias et al. (1967) described pronounced improvements in patients with Parkinsonism treated with the racemic mixture DL-dopa in doses of up to $16 \mathrm{~g}$. daily. Further studies by Cotzias et al. (1969a, 1969b), Duvoisin et al. (1969), and Yahr et al. (1968) showed that the laevo isomer L-dopa was an effective treatment of Parkinsonism, and that the dose required was half that of DLdopa, and that the danger of marrow depression was much less than with the latter drugs. Calne et al. (1969) showed that L-dopa is useful in the short-term treatment of patients with postencephalitis Parkinsonism. Godwin-Austen et al. (1969) also described improvement in patients with Parkinsonism treated with L-dopa for a few weeks. This study describes experiences of patients with Parkinsonism treated with L-dopa for several months.

\section{Patients}

Thirty-two patients (18 men and 14 women) were treated with L-dopa. Their ages ranged from 42 to 76 , average 61 years. Six patients were over 70 and four were less than 50 . Farkinsonian symptoms had been present for periods ranging from 3 to 47 years.

The likely aetiology of Parkinsonism was assessed in each case. Many causes are recognized, but it is generally accepted that most cases can be categorized under the three headings of postencephalitic, idiopathic (or paralysis agitans), and atherosclerotic, though the entity of atherosclerotic Parkinsonism has been disputed. It is sometimes difficult to place individual patients into one of these categories with certainty. Of the present group, six could confidently be labelled as postencephalitic on the basis of their histories and clinical features. The early age of onset of symptoms suggested an encephalitic aetiology in a further three patients, but the lack of confirmatory history and of characteristic signs leaves the diagnosis indeterminate in these patients. Most patients (24) were labelled idiopathic paralysis agitans, but in at least four and possibly in some of the other elderly patients cerebral atherosclerotic changes may well have played a part in producing their disabilities. Four patients had undergone previous surgical treatment for their Parkinsonism. There was no selection of patients because of age or severity of affection, but those who were demented or who gave a clear history of cerebrovascular disease were excluded from the trial. 


\section{Methods of Assessment}

The assessment of Parkinsonian disability and of improvement or change therein offers considerable difficulty. Three cardinal features of tremor, rigidity and hypokinesis comprise the Parkinsonian syndrome. Though for diagnostic purposes these three components are considered separately, their relative contributions to the motor deficit of Parkinsonism are not easily separable. Denny Brown (1949) emphasized the interrelationship between these features. He has shown that both tremor and rigidity represent contraction in the same muscle groups. His electromyographic studies showed that there was often a transition from rhythmically interrupted contractions representing tremor to the steady contraction of rigidity. There is a well-recognized clinical variability in tremor, dependent on such factors as anxiety and temperature, which makes grading of the severity of tremor difficult.

Impoverishment of movement, called akinesis or hypokinesis, was thought to be the result of muscular rigidity. Rigidity certainly impedes movement, but there is a lack of correlation between akinesis and the degree of associated rigidity which has long been recognized (Wilson, 1925). Operative treatment of Parkinsonism has shown that rigidity may be relieved and yet hypokinesis remain unchanged or, indeed, become more pronounced. Denny Brown (1968) thought that the primary symptom of damage to the basal ganglia is hypokinesis which he defined as difficulty in the initiation of willed movement. He pointed out that hypokinesis is not so much a loss of a particular category of movement as a defect of performance under general circumstances. It is apparent, therefore, that the critical evaluation of the relative importance of rigidity and hypokinesis is extremely difficult. Rigidity may be assessed and graded by the normal clinical manoeuvre of putting the limb through a series of passive movements and estimating the resistance to such movements offered by the limb. It is, however, difficult to grade, and compare at intervals, such resistance objectively. It is also difficult to separate the inertia of voluntary movements into the delay in initiation, and the slowing of movements once initiated.

Recognizing the inaccuracies and variabilities outlined above, the features of tremor, rigidity, and hypokinesis were graded respectively in each patient as "marked," "moderate," "slight," or "absent." All patients were assessed clinically before treatment, and repeatedly reassessed during the period of treatment. In addition to the three primary facets, the gait, the presence or absence of oculogyric crises, postural defects, impassivity of face, salivation, and mood were appraised. This clinical assessment was supplemented by recording on ciné film. Patients were filmed rising from a chair, walking, and performing fine finger movements before and during treatment. Specimens of handwriting were compared before treatment and while on treatment with L-dopa.

Hoehn and Yahr (1967) drew attention to the discrepancy between observable clinical signs and the degree of functional incapacity in patients with Parkinsonism. To evaluate the latter the assessment of clinical signs was supplemented by independent estimations of motor function made by physiotherapists and occupational therapists, who recorded their findings under standard headings and into four degrees of severity. All patients were graded on their ability to rise from a chair, to walk, to change direction, and to climb stairs. Patients' ability to perform such normal functions as dressing and feeding themselves was also ranked.

The patient's own impression of his progress was noted. Supplementary evidence about his performance at home was obtained from relatives and from his family doctor.

All patients were submitted to a series of timed dexterity tests. They were asked to perform a battery of manipulations-some were performed with each hand separately and some tested bimanual co-ordination. Readings before and during treatment enabled an objective timed assessment of manual dexterity to be made. The nature of these tests is shown in the Table.

Amplitude and rate of tremor were recorded electronically by Dr. Geoffrey Walsh. He subjected the wrist to oscillations of known force and rate with a torque generator. Spontaneous tremor and alterations therein, induced by the application of rhythmically varying torques, were recorded before treatment and again during treatment with L-dopa. The apparatus and methods used have been described by Walsh $(1968,1969)$, and the results of these investigations in this group of patients is to be published.

Speech was assessed clinically by the observing physician and recorded on tape by a speech therapist. The time taken for a patient to read a standard passage was noted. He then repeated eight vowel sounds. The recordings thus obtained were played through a condenser microphone, and the sound pressure level, which gives an index of voice volume, was measured in decibels. Average values of voice volume were obtained from the maximum and minimum sound pressure levels recorded during several 10 -second reading periods. By combining these results with the average value calculated from the vowel repetition an overall mean voice volume was computed. The figures thus obtained, though only approximate, give reasonably consistent results. Volumes between 65 and 70 decibels are normal, and a change in an individual of 3 decibels or more represents an appreciable alteration. In addition to the objective assessment of volume, speech therapists appraised the monotony of speech, the degree of dysarthria, delay in initiation of speech, and any tendency to undue acceleration when speaking. Recordings made before and during treatment in each patient were compared.

During the initial stages of treatment each patient's blood pressure was recorded in both erect and supine postures at least twice daily. Screening tests were performed on each patient before treatment, and repeated at intervals of about a fortnight throughout the period of treatment. Estimations of haemoglobin, counts of white cells and platelets, and liver function tests were regularly performed. The blood urea was estimated, and electrocardiographs were recorded.

Dexterity Tests in Case 14. Results Expressed in Seconds

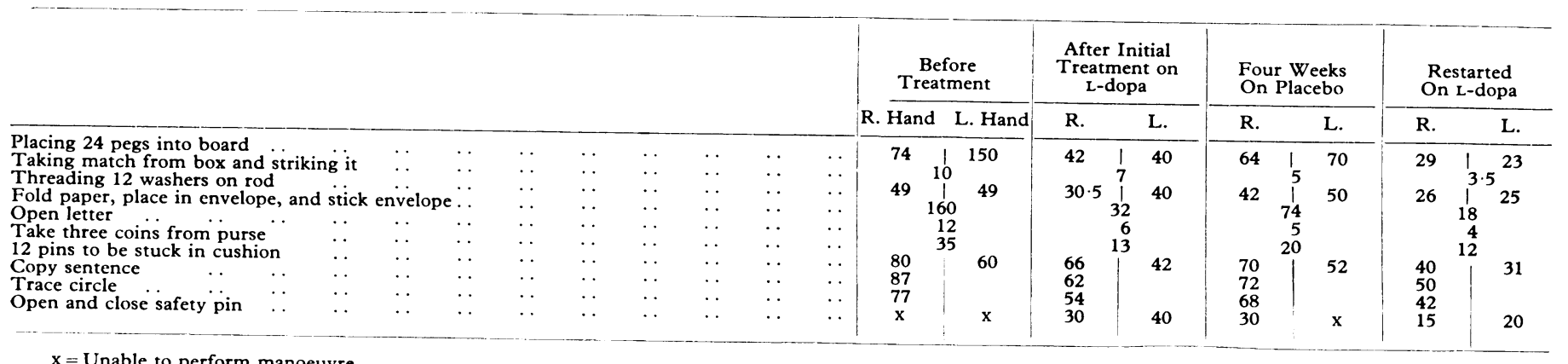




\section{Dosage and Administration}

All patients were initially admitted to hospital, and usually remained there for about four weeks. Thereafter, treatment was continued in the outpatient clinic, where patients were reviewed at fortnightly intervals. In all but three patients other anti-Parkinsonian drugs were stopped while L-dopa was given.

At the beginning of this study the drug used was given in freshly made doses from a bulk powder which was preserved in a water-tight container. The pharmaceutical stability and potency of this preparation could not be guaranteed. Seven patients in all were treated with this product for short periods. Later, a supply of stable L-dopa was obtained. This was supplied in capsules, each of which contained $150 \mathrm{mg}$. of Ldopa of a consistently high degree of purity (not less than $99 \%$ ). Recently, capsules containing $500 \mathrm{mg}$. of the drug have been available, and this is now the standard preparation used. Each patient was started on $0.9 \mathrm{~g}$. of $\mathrm{L}$-dopa daily, given in three divided doses. The drug was administered after meals to minimize nausea. The dose was increased by amounts of a further $0.9 \mathrm{~g}$. at intervals of three to four days. As the total daily dosage increased, the number of doses was also increased up to a maximum of eight daily. When nausea and vomiting were distressing to patients they were also given cyclizine $5 \mathrm{mg}$. thrice daily.

The increments of L-dopa were continued until an adequate therapeutic response was achieved, or until troublesome side-effects supervened. When patients developed distressing side-effects treatment sometimes had to be discontinued, otherwise the dose was reduced to the highest tolerated level and patients were then treated for an initial period of six weeks. If no significant improvement was seen at the end of this time, the treatment was discontinued. When a worthwhile response was obtained L-dopa was continued. The effective daily dose varied between 1.5 and $9 \mathrm{~g}$. At the time of discharge from hospital most patients were taking between 4 and $6 \mathrm{~g}$. daily.

Twenty patients were included in a double-blind "crossover" trial. Patients at random were given a placebo for periods varying from two to six weeks. The placebo was identical in form to the active drug, and neither patient nor observing physician was aware of the drug received. Though all patien's were aware that they were taking part in a trial of the new drug none was told that he might be given $n$ inact:ve preparation during the period of treatment.

\section{Results}

Twenty patients who tolerated the drug and who derived significant benefit from it were treated for an average period of six months. The minimum duration of treatment in this group was two months (two patients). Eighteen patients received L-dopa for more than three months and five of these were treated for at least six months, five others for nine months, and two for almost a year. In 12 patients treatment was discontinued, for reasons analysed below, after periods varying from two to six weeks.

In each patient an overall assessment of benefit was estimated. We found that only this overall evaluation gives an adequate index of therapeutic response in Parkinsonism. As Hoehn and Yahr (1969) pointed out, the amount of disability arising from the neurological abnormality of Parkinsonism often depends on patients' values and needs. The relative import of such features as a defect in gait, deficits in fine finger movements, and speech impairment varies widely from individual to individual. Thus we believe that the use of an index of disability achieved by summation of the ran'zings of various criteria does not adequately reflect either the degree of disability or the change therein. In each case we assessed all the clinical features and objective criteria listed above, and graded the patients' responses as "marked," "moderate," "slight," or "none."

\section{Marked Improvement}

Nine patients were considered to be markedly improved. Improvement was considered marked in those patients who before treatment had impairment of motor function or speech conspicuous enough to interfere with the activities of daily living, and who after treatment were independent in such activities. Such marked improvement does not necessarily imply restoration to normality, nor the abolition of all signs of the disease but rather a restoration of function to a socially and economically acceptable level.

Two patients achieved virtual normality after treatment which in each case was continued for more than six months. In the remaining seven patients the features of Parkinsonism, though greatly ameliorated, were still obvious. Though these nine patients were strikingly improved, the nature and quality of the improvement varied. This variation may best be illustrated by two examples.

\section{Case 14}

A woman aged 61 gave a 10-year history of tremor affecting both arms, more prominent on the right side. For the same time there had been a gradually progressive slowness and stiffness of her gait. Poverty of movement became more prominent, though her tremor was less evident. She began to fall frequently so that on two occasions during the previous three years she had beer admitted to hospital for periods of several weeks, during which time various combinations of drugs were tried and she received intensive physiotherapy. No perceptible improvement occurred. For three years she had been unable to turn in bed or, unaided, to get out of bed. Her husband did the housework and shopping, and had to wash, dress, and feed her. She was unable to walk to the lavatory without his support, and even when helped to walk she often fell.

She was readmitted to hospital 11 months ago. Clinical examination showed marked imoassivity of features and profound rigidity and akinesis which affected all four limbs, especially on the right side. She had to be lifted from her bed to a chair, and was unable to rise from a chair unaided, and even with the support of two helpers was unable to walk. Dexterity tests showed profound imoairment. The results obtaired at this time are listed in the Table. Her speech was slow and monotonous but clearly in telligible, and her voice volume fell within the normal range at 66 decibels. After assessment in hospital and discontinuation of anti cholinergic drugs she was started on L-dopa. The dosage was gradually increased, so that after four weeks she was taking $5.4 \mathrm{~g}$ daily. On this dose she developed troublesome oral dyskinesis and alternating flexion and extension movements of her feet, particularly obvious in the left foot. The dose was therefore reduced to $4.5 \mathrm{~g}$. dailv, and on this dosage she was discharged. At this time she was able to rise from a chair unaided and to walk fairly easily with the aid of one stick. Her dexterity tests showed considerable improvement (see Table). She continued thus for a further two months, at the end of which time she was given a placebo, the number of capsules remaining unaltered. After two weeks she complained, on a visit to the outpatient department, of gradually increasing disability, and after four weeks on the placebo she was referred back to hospital by her family doctor, who asked for her admission as an urgency. In his referral letter he said that since she left hospital she had been "a completely different woman, alert and walking much better, but she had now rapidly deteriorated and was again unable to walk."

At the time of her readmission all the previously noted clinical findings were present, though these were graded as slightly less marked than they were before treatment started. Results of dexterity tests at this time are given in the Table. She was again unable to rise from a sitting posture, and was able to walk only for very short distances and very slowly even with the help and support of the physiotherapist. She was again started on an active preparation, and again the dose was increased until she was taking $4.5 \mathrm{~g}$. daily in $\mathrm{s}: \mathrm{x}$ divided doses. Once more she improved, was able to walk without a stick or other support, and dexterity tests showed significant improvement. She returned home and continued on L-dopa. On two occasions she developed oral dyskinesis, and 
the dose was reduced so that for the past two months she had been taking only $2 \mathrm{~g}$. of L-dopa daily. At the time of writing she was able to dress herself, climb stairs to her bedroom, and attend to her own toilet. She is able to do the bulk of the family's housework and washing, and does her own shopping. Speech shows a perceptible improvement. She speaks more rapidly, and there has been an increase in her voice volume to 68 decibels.

\section{Case 18}

This patient, a man aged 50, had suffered an attack of encephalitis 29 years ago while a prisoner-of-war. His consciousness was clouded for three to four weeks before admission, and he has a complete amnesia for this period. Immediately after recovery from this illness he developed marked difficulty with his speech which persisted. In addition he suffered from attacks wherein his eyes deviated conjugately, usually to the right, but occasionally upwards. Such attacks lasted for several hours, and recurred at intervals of two to three weeks since the time of their onset.

On examination he showed facial impassivity, and salivated profusely. He had slight impairment of fine movements in both hands. There was absence of associated movements when he walked, though his gait was virtually normal. Dexterity tests were performed normally. His handwriting, though legible, was micrographic. The most striking clinical abnormality was the speech impairment. His greatest disability was in the initiation of speech. There would be long periods when he would attempt to speak and be unable to phonate at all. When he did begin to speak he was dysarthric and, though initially there was bradylalia, he would of ten rapidly accelerate his speech so that it became quite incomprehensible; then it would abruptly stop and again would follow a long period during which he could not phonate. The cadence of his speech mimicked the disabilities more commonly seen in the gait of patients with Parkinsonism. Clinically his speech was reduced in volume, and this was confirmed by a voicevolume recording which gave an average value of 58 decibels before treatment.

He was started on L-dopa, achieving a maximum dose of $4.5 \mathrm{~g}$. daily after four weeks. After a month, his speech showed striking improvement. It was obviously louder, and his average voice volume was 64 decibels, which represents a highly significant degree of improvement. His dysarthria was greatly reduced, and the profound difficulty in initiating speech virtually disappeared. His handwriting increased to normal size, and his dexterity tests showed minor improvements. He continued on treatment for six months, though during this time he had a period of four weeks when he was taking the inactive preparation. During the period on placebo his speech deteriorated, and this was obvious both to him and to the observing clinician, but even after four weeks it did not regress to the profound impairment which was present before treatment started. His speech remained improved. Since starting on L-dopa he had suffered no further oculogyric crises. His salivation was much less evident. He continued to work as a labourer, and was relieved of a previously embarrassing difficulty when talking with his workmates.

Both patients showed improvement which qualitatively was very different but which in each case was manifest to a marked degree. In Case 14 the slight improvement in speech was irrelevant where in Case 18 improvement in speech and abolition of oculogyric crises profoundly affected the patient's efficiency, and the minor gain in motor function was to him insignificant. These examples illustrate the need for a flexible method of assessment in patients with Parkinsonism.

\section{Moderate Improvement}

Fourteen patients showed moderate improvement. Improvement was graded as moderate in those patients who showed considerable improvement in gait, in motor function, or in speech accompanied by significant change on the objective tests, but who yet still had residual disability and needed help in the activities of daily life.

Twelve patients of this group continued to take L-dopa. Two who were moderately improved have discontinued treatment. The first of these, a women with postencephalitic Parkinsonism, developed lingual dyskinesia on low dosage, and preferred her previous motor disability to this side- effect. The other patient, one of the oldest in the group, was considerably improved by L-dopa in high dosage, but suffered from postural hypotension and was still so disabled that he needed hospital inpatient care; it was felt, therefore, that treatment was not beneficial enough to justify its continuation.

\section{Slight Improvement}

Five patients came under this category. Improvement was regarded as slight in those who showed a perceptible improvement in their disabilities but in whom the improvement did not greatly alter their functional capacity. In these patients the amelioration of symptoms was no greater than that obtained by treatment with anticholinergic drugs, encouragement, and physiotherapy.

\section{No Improvement}

No improvement was discernible in five patients, but in two of these the drug was discontinued after only two and four weeks, respectively, because of profound side-effects.

\section{Effects on Clinical Features of Parkinsonism}

The earliest beneficial effect of L-dopa was manifest by a lightening of mood and brightening of eye which was obvious in most patients within 10 days of starting treatment. This elevation of mood was exaggerated to the point of euphoria in three patients. Early feelings of well-being often led to assertions of improvement by patients in whom there was no discernible clinical change, and this increased the difficulties of objective assessment.

In those who were helped by L-dopa increased motor facility was the first perceptible change in physical signs. Relief of hypokinetic disabilities was evinced early, usually after two to three weeks of treatment, and was enhanced in the ensuing six weeks. All the clinical features which reflect the basic hypokinetic defect shared in the improvement to some extent. Facial expression became more animated, gait was improved, manipulation of the hands became more dexterous, and speech increased in volume, varied in pitch, and approached normal rhythm and cadence. The most potent and consistent therapeutic effect of $\mathbf{L}$-dopa was the relief of hypokinesis.

Rigidity, too, was ameliorated, to a degree and at a rate which usually lagged behind the improvement of hypokinesis.

Diminution of tremor was neither conspicuous nor frequent during the period of treatment of this group of patients. Two patients whose tremor was graded initially as moderate and slight respectively, now have no visible tremor. The decrease in amplitude of the tremor in these cases was gradual, and the tremor was abolished only after several months' treatment. Recordings of the tremor in one of these patients are shown in Figs. 1 and 2. Absence of tremor for such a short period as is
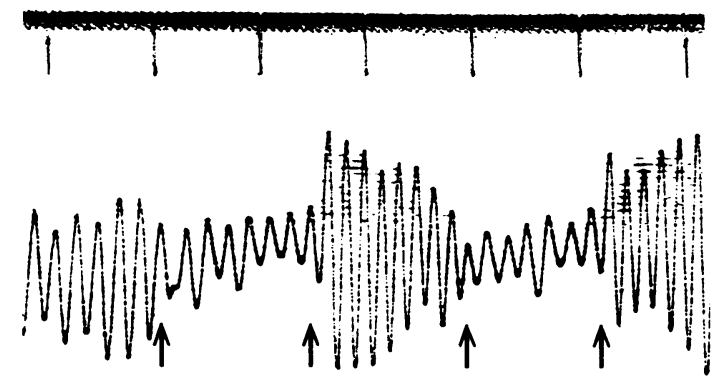

FIG. 1.-Case 15. Recording from right wrist before treatment. Periods between the first two arrows and between the third and fourth arrows show spontaneous $5 \mathrm{c}$./ $\mathrm{sec}$. tremor. Larger amplitude deflections enclosing these periods result from an applied torque. 

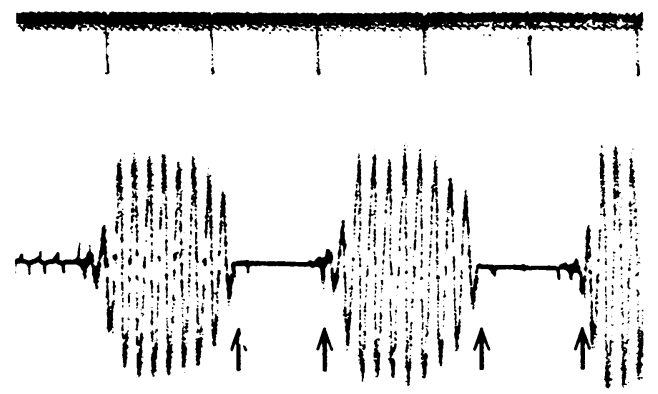

FIG. 2.-Case 15. Corresponding reading after four months' treatment. Flat baseline between first and second arrows and between third and fourth arrows shows disappearance of spontaneous tremor. The interspersed bursts of deflections result from an applied torque. Time scale marked in seconds. Calibration $200^{\circ} / \mathrm{sec}$.

recorded in Fig. 2 does not, of course, confirm its disappearance; but this is a representative sample from a long recording session, and tremor has not been clinically discernible during her visits to th eoutpatien tclinic in recent months.

Five other patients treated with L-dopa for more than three months have shown an apparent decrease in amplitude and less frequent occurrence of tremor. These observations are of necessity rather tentative, since repeated short periods of observation in an outpatient clinic do not constitute a firm basis for attribution of improvement in such a notoriously variable phenomenon. Tremor is aggravated by anxiety and often is increased in the unfamiliar surroundings of a hospital. After repeated visits to the same physician in the same clinic patients would be expected to be less intimidated by their visits, and this alone would account for some apparent lessening of tremor. No convincing improvement in tremor was observed in the remaining patients in the group.

In four patients an increase in the amplitude of tremor was observed. This usually occurred after four to six weeks of treatment with L-dopa, and probably reflects lessening of rigidity. The change from a sustained contraction of muscle groups to the intermittent contractions of tremor would thus represent a stage of improvement. This accords well with Denny Brown's concepts outlined above, and is supported by the readings shown in Figs. 3 and 4. This man had no tremor
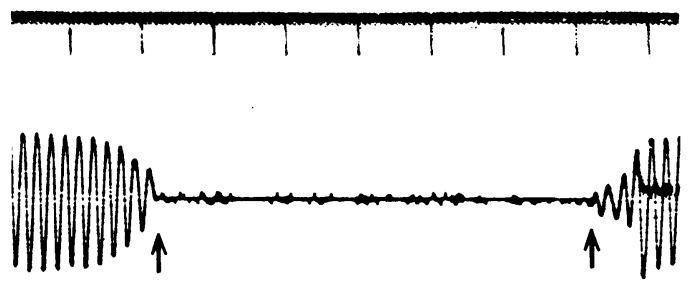

Fig. 3.-Case 9. Recording from right wrist before treatment. Flat baseline between arrows shows absence of spontaneous tremor. Periods before first arrow and after second arrow result from applied torque.
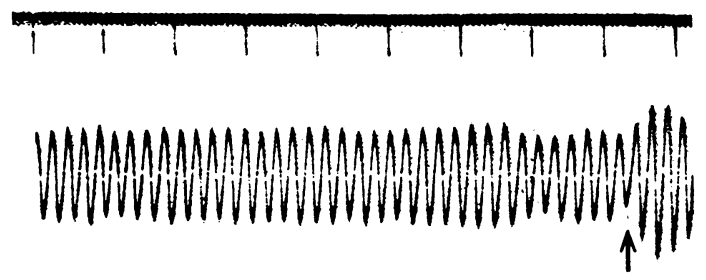
FIG. 4.- Case 9. Corresponding recording after four weeks treatment. Arrow shows beginning of period of
applied torque. Period to the left of arrow is spontaneous applied torque. Period to the left of arrow is spontaneous
tremor, rate about 4 c./sec. Time scale marked in
seconds. Calibration $200^{\circ} / \mathrm{sec}$. discernible clinically, nor electronically before treatment with L-dopa (Fig. 3). He did exhibit marked rigidity. After four weeks on L-dopa he developed tremor (Fig. 4). In this case the side-effects of the drug caused the patient's decision to discontinue L-dopa shortly after the second recording; therefore, we could not investigate the later effects of the drug. In the remaining patients who exhibited an exacerbation of tremor this apparent aggravation of symptoms was temporary and persisted for only two to three weeks. This transient phenomenon, which may distress patients who regard it as deterioration, does not constitute grounds for stopping the drug.

Three patients who suffered from frequent oculogyric crises were completely free of these while on L-dopa. One of them had a recurrence five weeks after stopping the drug.

Only four patients in this series had undergone previous surgery; two responded moderately well and two poorly. It is of interest that, of these four, the three who had paralysis agitans developed no side-effects on high doses, one patient taking $9 \mathrm{~g}$. daily without any evidence of induced involuntary movements.

The improvement in these patients was sustained over an average period of six months, and at least three patients increasingly benefited from treatment throughout a six-month period.

\section{Double-blind Trial}

Twenty patients took part in an attempted "double-blind" trial. Four of these received the inactive preparation for two to six weeks at the start of treatment, the capsules being increased in number and the patients being assessed and given physiotherapy in the usual way. One of these was thought to show slight improvement while on the inactive capsules, but his incapacity was still so severe that it was decided to stop treatment after six weeks. He has not yet received the active drug, but is shortly being admitted so that his response to Ldopa may be assessed. He thought that he deteriorated after the placebo was discontinued. No improvement was observed in the other three patients while on the placebo, though later treatment with L-dopa produced improvement which was graded as moderate in two and slight in the other.

Sixteen patients who had initially received L-dopa and had shown a worth-while response were "crossed over" to the placebo for two to four weeks, after periods of active treatment varying from four weeks to four months.

Six patients took the placebo for only two weeks and appreciable deterioration was observed in two of these. In all the remaining 10 appreciable deterioration was observed and noted after four weeks on the inactive preparation. In six of these patients profound disablement recurred, and four were readmitted to hospital because of immobility. In the ward the active preparation was reintroduced in gradually increasing dosage, and in all the patients the previously attained gains were restored after three to four weeks.

Patients who were abruptly restarted on effective doses of L-dopa usually had a resurgence of vomiting, which was often much more severe than during the initial treatment phase. In three patients sudden reinstitution of the active preparation in high dosage caused such profound nausea, vomiting, and weakness that they were confined to bed for several days. In these three patients the daily dose of L-dopa was drastically reduced, and thereafter gradually increased to the previously effective level.

Clearly these profound effects led to virtually complete decoding by the observing physician. In three instances the patients, too, though given no forewarning, deduced that the placebo capsules were an ineffective substitute for the drug. Those who did not make this deduction were usually aware that they had regressed after a month on the placebo and 
decided that the drug had lost its effect and several became depressed. Only two patients were unaware of their observed deterioration after a month on inactive capsules. One of these did not suffer from vomiting when L-dopa was reintroduced, and he was given a further period on the placebo after an interval of effective treatment with similar objective deterioration without subjective recognition thereof. It had been intended to extend the trial over a prolonged period with repeated "cross over," but the distressing effects enumerated above led us to terminate this trial.

Abrupt cessation of L-dopa does not produce equally prompt deterioration, and the beneficial effects of the drug usually persist for two weeks and then progressive disability recurs; but complete regression to the pretreatment level of disability may not occur until more than four weeks after stopping treatment.

Extensive "decoding" by observers and patients occurred in this trial, as it did in that of Calne et al. (1969). The trial design was modified to meet the clinical needs of those patients who were readmitted to hospital, and in those treated at home whose vomiting led to adjustment of the dose of Ldopa. Valid "double-blind" conditions were not achieved, and it would be unrealistic to apply statistical techniques to the results. Nevertheless, the demonstration of the ill effects of substituting an inactive preparation for L-dopa at least indicates that the latter has a therapeutic effect and that its efficacy in Parkinsonism is not attributable to optimism, suggestion, or enthusiasm arising from its novelty.

\section{Side-effects}

Seven patients suffered no ill effects while taking the drug. Nausea and anorexia were the commonest side-effects. Two-thirds of the patients (22) complained of nausea at some stage during their treatment, and this was accompanied by vomiting in seven. It was usually possible to minimize nausea by giving cyclizine, by reducing the individual doses of L-dopa and giving these after food, or by reducing the total daily dose. In six patients nausea was one factor leading to the stoppage of treatment, but in only two was vomiting the principal reason for discontinuing the drug.

Induced involuntary movements were common. No patient developed true choreiform movements. The commonest and usually the earliest dyskinsias were stereotyped grimacing movements of the mouth, chewing movements of the jaws, or rotatary movements of the tongue. Often these oral dyskinesias were accompanied by alternating flexion and extension at the ankles, producing "paddling" foot movements. Where Parkinsonian symptoms were asymmetrical the involuntary foot movements usually began and were always more prominent on the less-affected side. Ten patients exhibited these relatively mild involuntary movements, which were never distressing. Two of these patients also developed spasmodic torticollis, which they found disturbing. One postencephalitic patient, treated for only two weeks, had taken $3 \mathrm{~g}$. of L-dopa for one day and on the following day developed severe painful dystonic spasms of the neck and trunk. These distressed her greatly, and treatment was discontinued. Involuntary movements invariably regressed or disappeared when the dose of L-dopa was reduced.

Six patients showed significant postural hypotension during the first few weeks of treatment. Two of these complained of faintness and dizziness. One of the asymptomatic patients derived little benefit after six weeks' treatment on L-dopa, which was stopped. Two weeks later he had a myocardial infarction. The relation between the infarct and his earlier postural hypotension is uncertain, but clearly there is at least a potential danger from postural hypotension in those patients who suffer from cerebrovascular or coronary arterial disease.
Psychiatric disturbances occurred. Three patients complained of mild depression during the early stages of treatment, but this persisted for only a few days. Anxiety, agitation, and irritability were manifested by two patients after several weeks on the drug, but these features regressed without reduction of dosage. Two patients developed frightening hallucinations. One was a man with a history of previous psychiatric illness who developed hallucinations and paranoid delusions after only two weeks on treatment, and refused to take any more capsules. After a few days these acute symptoms disappeared. The other patient, a woman, nad been treated for four months before she developed frightening hallucinations. These rapidly disappeared after a slight reduction in dosage. One other patient with postencephalitic Parkinsonism was markedly improved after six weeks' treatment; but at home she was extremely aggressive and began to swear compulsively and obscenely. She was distressed by this symptom, which rapidly regressed when her dose of L-dopa was slightly reduced. Two men and one woman noticed an increase in libido while taking the drug.

One patient developed a deep calf vein thrombosis after three months' treatment. Another suffered from frequent and severe headaches during the initial phase of treatment. One woman, not included in this series, developed an irritant widespread urticarial rash a few hours after taking her second capsule of $\mathrm{L}$-dopa. She was taking no other drug. I-Dopa was discontinued for a week, during which time she was given an antihistamine and the rash disappeared. After a week, while still taking the antihistamine, she was given another capsule of L-dopa and again, within hours, an urticarial eruption resulted. It seemed that she had had a hypersensitivity reaction to L-dopa, and treatment was abandoned. During the period of observation no patient has shown any appreciable change in his blood count, nor have liver function tests revealed any abnormality.

\section{Observations on Dosage}

Almost all patients who had been treated for more than three months are now taking a dose lower than their maximal tolerated dosage. After several months' treatment many patients developed dyskinesias on doses which previously had produced no side-effects. These regressed after reduction in dosage without any detectable deterioration in motor function. Most patients who had been treated for more than six months are taking 2 to $4 \mathrm{~g}$. of L-dopa daily, which is about half the amount these same patients took at the end of the six-weeks initial treatment phase. Cotzias et al. (1969a) has commented on the therapeutic response which is maintained as the dosage is lowered in patients on prolonged treatment. Cotzias et al. (1969a) have also stressed the importance of a gradual increase in the dose of L-dopa and the need to reduce the daily increments, sometimes to as little as $50 \mathrm{mg}$. in order to minimize undesirable side-effects. With this in mind, we have restarted on L-dopa three patients who developed sideeffects on a low dose early in the course of the original attempt at treatment. The initial dose was reduced to $300 \mathrm{mg}$., and increments of $150 \mathrm{mg}$. daily were added at weekly intervals. All three patients are now taking, without ill effect, a daily dose of L-dopa substantially greater than that which previously produced distressing side-effects. It is too early to assess the therapeutic effects in these three patients whose dosage continues to be augmented.

\section{Discussion}

Twenty of this group of 32 patients tolerated prolonged treatment with L-dopa and derived appreciable improvement 
from it. Nine patients $(28 \%)$ were markedly improved. These results are roughly comparable to those of Yahr et al. (1968) and Cotzias et al. (1969a).

It is probable that some of the 12 patients in this series who derived slight benefit, or none, from L-dopa, or whose treatment was curtailed because of ill effects, might be helped by the drug if a more gradual build-up of the dose was used, as suggested by Cotzias et al. (1969a). It is probable, too, that treatment on the maximal tolerated dose should be prolonged for at least six months before presuming that L-dopa is ineffective in a particular patient.

Improvements in hypokinesis and rigidity were much more striking in this group than was amelioration of tremor. Cotzias et al. (1969a) indicated that tremor is the last feature to show improvement, and it may be that continuing treatment of our patients will result in more striking relief of tremor.

It is not possible to predict with certainty which Parkinsonian patients will derive most benefit from L-dopa treatment. There is no consistent correlation with age, with duration, or with severity of disease. The few patients in this series who had postencephalitic Parkinsonism were prone to develop unpleasant side-effects on low doses-which is consistent with the observations of Calne et al. (1969). It seems probable that a very gradual and prolonged build-up of the drug should be adopted in postencephalitic patients.

Most patients have some untoward side-effects at some stage while taking L-dopa. A substantial number of people suffer unpleasant or distressing reactions, but all the sideeffects observed in this series were dose-dependent and readily reversible. Postural hypotension seems to be the only potentially dangerous complication of treatment. Further observations of large numbers of patients for periods of years are needed before the safety of L-dopa can be fully established.

There is now strong evidence that L-dopa offers a most effective treatment of Parkinsonism. It improves function for long periods, but it has yet to be shown whether the improvement produced is sustained indefinitely, or whether the drug alters the natural history of this disease.

I am grateful to my colleagues Dr. J. B. Stanton and Dr. E. H. Jellinek for permission to study patients under their care and to Dr. D. R. Wood for his help in running the trial. To Dr. Geoffrey
Walsh I am indebted for his physiological studies on these patients, for the illustrations of tremor, and for much helpful discussion. I would like to acknowledge the help received from Miss Mary Harper, Miss Christine Allan, and Miss Irene Nash, who carried out many of the assessments in these patients. To Dr. $\mathrm{H}$. Holgate and Roche Products Limited I am particularly grateful for generously supplying the L-dopa used in this study.

\section{REFERENCES}

Barbeau, A. (1962). Canadian Medical Association fournal, 87, 802. Birkmayer, W., and Hornykiewicz, O. (1961). Wiener klinische Wochenschrift, 73, 787.

Bloom, F. E., Costa, E., and Salmoiraghi, G. C. (1965). fournal of Pharmacology and Experimental Therapeutics, 150, 244.

Calne, D. B., Stern, G. M., Laurence, D. R., Sharkey, J., and Armitage, P. (1969). Lancet, 1, 744.

Carlsson, A. (1959). Pharmacological Reviews, 11, 490

Carpenter, M. B., and McMasters, R. E. (1964). American fournal of Anatomy, 144, 293.

Cotzias, G. C., Papavasiliou, P. S., and Gellene, R. (1969a). New England fournal of Medicine, 280, 337.

Cotzias, G. C., Papavasiliou, P. S., Gellene, R. Aronson, R. B., and Mena, I. (1969b). In Third Symposium on Parkinson's Disease, edited by F. J. Gillingham and I. M. L. Donaldson, p. 178. Edinburgh, Livingstone.

Cotzias, G. C., van Woert, M. H., and Schiffer, L. M. (1967). New England fournal of Medicine, 276, 374

Denny-Brown, D. (1949). Archives of Neurology and Psychiatry, 61, 99.

Dennv-Brown, D. (1968). In Handbook of Clinical Neurology, vol 6 , Diseases of the Basal Ganglia, edited by P. J. Vinken and G. W. Bruyn, p. 133. Amsterdam, North Holland Publishing Company.

Duvoisin, R., Barrett, R., Schear, M., Hoehn, M., and Yahr, M. (1969). In Third Symposium on Parkinson's Disease, edited by F. J. Gillingham and I. M. L. Donaldson, p. 185. Edinburgh, Livingstone.

Ehringer, H., and Hornykiewicz, O. (1960). Klinische Wochenschrift, 38 1236 .

Fehling, C. (1966). Acta Neurologica Scandinavica, 42, 367.

Godwin-Austen, R. B., Tomlinson, E. B., Frears, C. C., and Kok, H. W. L. (1969). Lancet, 2, 165.

Greer, M., and Williams, C. M. (1963). Neurology (Minneapolis), 13, 73.

Hassler, R. (1938). Fournal für Psychologie und Neurologie, 48, 387 .

Hoehn, M. M., and Yahr, M. D. (1967). Neurology (Minneapolis), 17, 427.

Hoehn, M. M., and Yahr, M. D. (1969). In Third Symposium on Parkinson's Disease, edited by F. J. Gillingham and I. M. L. Donaldson, p. 274. Edinburgh, Livingstone.

Rinne, U. K., and Sonninen, V. (1968). European Neurology, 1, 180.

Rundles, R. W., and Papez, J. W. (1937). Archives of Neurology and Psychiatry, 38, 550.

Trétiakoff, C. (1919). Contribution à l'étude de l'anatomie pathologique du Locus niger de Soemmering. Thesis, Paris.

Vogt, M. (1954). Yournal of Physiology, 123, 451.

Walsh, E. G. (1968). Fournal of Physiology, 198, 69P

Walsh, E. G. (1969). Ұournal of Physiology, 202, $109 \mathrm{P}$

Wilson, S. A. K. (1925). Lancet, 2, 53.

Yahr, M. D., Duvoisin, Lancet, $2,53$.

, M. D., Duvoisin, R. C., Hoehn, M. M., Schear, M. J., and Barrett, R. E. (1968). Transactions of the American Neurological As-
sociation, 93, 56.

*Physician, Royal Northern Hospital, London N.7. tPfizer Research Fellow.

†Biochemist, Royal Northern Hospital, London N.7

SSupported by research grant from North-West Metropolitan Regional Hospital Board.

\section{Introduction}

It is generally accepted that adrenal function is reduced in primary hypothyroidism. This has been attributed both to an inadequate response of the adrenal cortex to corticotrophin (Macgregor, 1964) and to an impairment of pituitary function (Lessof et al., 1969). In myxoedema coma hydrocortisone is recommended because of the likelihood of associated adrenocortical insufficiency (Ingbar and Woeber, 1968). There is, however, considerable disagreement among those who have studied this problem. The cortisol secretion rate is known to be reduced in myxoedema (Cope, 1964); this is associated with a normal plasma corticosteroid level and a reduced excretion of urinary hydroxycorticosteroids (Levin and Daughaday, 1955). 